\title{
Caracterización de las quemaduras por sustancias químicas en el Hospital Universitario de Santander, Bucaramanga, Colombia, entre 2009 y 2014
}

\author{
Sandra Liliana Jaimes Osorio* \\ Mónica Alexandra Ramírez Blanco** \\ Carlos Enrique Ramírez Rivero***
}

\begin{abstract}
*Médica y cirujana. Médica asistencial servicio de cirugía plástica, reconstructiva y estética. Hospital Universitario de Santander. Bucaramanga. Santander. Colombia.

**Residente de tercer año especialización en cirugía plástica, reconstructiva y estética. Universidad Industrial de Santander. Bucaramanga. Colombia. ***Especialista en cirugía plástica, reconstructiva y estética. Jefe de la Unidad de Quemados del Hospital Universitario de Santander. Docente y coordinador del postgrado de cirugía plástica, reconstructiva y estética. Universidad Industrial de Santander. Bucaramanga. Santander. Colombia. Correspondencia: Dra. Sandra Liliana Jaimes Osorio. Dirección: carrera 30 No. 31-66, piso 501. Bucaramanga. Santander. Colombia. Correo electrónico: salejaos_1906@hotmail.com
\end{abstract}

\section{RESUMEN}

Introducción: las quemaduras por sustancias químicas son consideradas lesiones graves por su alto potencial de causar daño local y sistémico, representan entre el 2,4\% y el 10,7\% de los pacientes admitidos en las unidades de quemados, con una mortalidad asociada del 30\%. La literatura disponible en Latinoamérica es escasa. Objetivo: el objetivo del estudio es determinar las características clínicas y epidemiológicas de los pacientes con quemaduras químicas atendidos en la Unidad de Quemados del Hospital Universitario de Santander, Bucaramanga, Colombia. Materiales y métodos: se realizó un estudio de tipo observacional, descriptivo, retrospectivo, en el cual se incluyeron los pacientes que presentaron quemaduras por agentes químicos entre el 1 de enero de 2009 hasta 31 de diciembre de 2014; se incluyeron 29 pacientes. Resultados: las quemaduras químicas representaron el 1,5\% de todas las quemaduras; predominio en el género masculino 17 casos; todas con una profundidad grado II y III; las extensiones no superaron el $25 \%$ de la superficie corporal total; el sitio anatómico más comprometido fue el miembro superior; hubo mayor asociación con accidentes de tipo laboral con 16 casos; los ácidos como el principal agente químico implicado con 17 casos y 5 lesiones por agresión; 19 ameritaron manejo quirúrgico. Conclusiones: las quemaduras por sustancias químicas representan un porcentaje pequeño pero significativo de las quemaduras, existiendo una clara asociación a situaciones accidentales por falta de capacitación en la manipulación adecuada de sustancias químicas, lo que plantea la necesidad de tomar medidas de prevención primaria. MÉD.UIS. 2016;29(1):11-6.

Palabras clave: Epidemiología. Quemaduras químicas. Unidad de quemados.

\section{Characterization of chemical burns at the Hospital Universitario de Santander, Bucaramanga, Colombia, between 2009 and 2014}

\section{ABSTRACT}

Introduction: chemical Burns are serious injuries due to their high potential to cause local and systemic damage. They represent between $2.4 \%$ and $10.7 \%$ of the patients admitted to burn units, and have an associated mortality of $30 \%$. The available literature on Latin America is scarce. Objective: the main objective of the study is to determine the epidemiological and clinical characteristics of patients with chemical burns treated at Hospital Universitario de Santander burn unit, in Bucaramanga, Colombia. Methods: this was an observational, descriptive, and retrospective study, which included patients who presented with burns by chemical agents between January 1,2009 to December 31, 2014; 29 patients were included. Results: chemical burns accounted for 1.5\% of all burns; 17 cases were male gender; all were II and III degree burns; total body surface did not exceed $25 \%$. The most affected site was the upper limb; there was a greater association with work related accidents with 16 cases; acids were the principal chemical agent involved with 17 cases and there was encountered 5 
injuries by assault; 19 patients required surgical intervention. Conclusions: chemical burns represent a small but significant percentage of burns, there is a clear association to accidental situations due to lack of training in the proper handling of chemicals, raising the need for primary prevention measures. MÉD.UIS. 2016;29(1):11-6.

Keywords: Epidemiology. Chemical burns. Burn Unit.

¿Cómo citar este artículo?: Jaimes SL, Ramírez MA, Ramírez CE. Caracterización de las quemaduras por sustancias químicas en el Hospital Universitario de Santander, Bucaramanga, Colombia, entre 2009 y 2014. MÉD.UIS. 2016;29(1):11-6.

\section{INTRODUCCIÓN}

Las quemaduras se clasifican de acuerdo a su profundidad en quemaduras de primer, segundo y tercer grado. Las de primer grado comprometen únicamente la epidermis; las de segundo grado comprometen la totalidad de la epidermis y parcialmente la dermis, reepitelizan a partir de los anexos cutáneos como glándulas sebáceas, glándulas sudoríparas y folículos; y las de tercer grado comprometen completamente la dermis y por tanto no pueden reepitelizar a partir de los anexos cutáneos por lo cual siempre ameritan manejo quirúrgico con injertos o colgajos. Las quemaduras por agentes químicos corresponden a quemaduras grado dos y tres en la mayoría de los casos, pero con un muy alto riego de progresión local y sistémica por absorción desde la piel, por lo cual se consideran graves sin importar su extensión'.

Son más de 25000 los agentes químicos utilizados en la industria y en el hogar que pueden generar quemaduras graves en la piel, y quienes manipulan este tipo de sustancias generalmente no tienen conocimiento del potencial daño o lo subestiman². Estas representan una pequeña pero significativa proporción de los pacientes que ingresan a las unidades de quemados con incidencias reportadas entre el $2,4 \%$ y el $10,7 \%$, con una mortalidad asociada de hasta el $30 \% 3$. Pese a su importancia, la literatura disponible en Latinoamérica es escasa y la mayoría de estudios recientes se han enfocado en quemaduras químicas asociadas a lesiones por agresión dado el incremento de esta problemática social en países en vía de desarrollo, desconociéndose la ocurrencia general y características clínicas de este tipo de lesiones.

La unidad de quemados del Hospital Universitario de Santander(HUS), Bucaramanga, Colombia, es el único centro de referencia para la atención de pacientes quemados con criterios de manejo intrahospitalario del nororiente colombiano. Esta dependencia del HUS tiene un área de influencia que abarca los departamentos de Santander, Norte de Santander, Sur de Bolívar, Sur del Cesar y Arauca, con un total aproximado de 4 millones de habitantes. Cuenta con 21 camas disponibles, y atiende tanto a régimen contributivo como subsidiado. Son admitidos un promedio de 324 pacientes nuevos por año ${ }^{4}$.

El objetivo de este estudio es determinar la características clínicas y epidemiológicas asociadas a las quemaduras químicas de los pacientes admitidos en la Unidad de Quemados del HUS en el periodo comprendido entre el año 2009 al 2014, lo que puede contribuir al desarrollo de futuras guías de manejo y protocolos para prevención primaria basados en estadísticas propias.

\section{Materiales y Métodos}

Se llevó a cabo un estudio de tipo observacional, descriptivo y retrospectivo, en el cual se incluyeron todos los pacientes que presentaron quemaduras por agentes químicos entre el 1 de enero de 2009 hasta 31 de diciembre de 2014. La obtención de los datos se realizó mediante la base estadística de la Unidad de Quemados del HUS. En esta unidad se admiten todos los pacientes en los que se documente quemadura de etiología química sin tener en cuenta su profundidad ni extensión, dado que son clasificadas desde el inicio como quemaduras graves y siempre ameritan manejo intrahospitalario'. La profundidad de la quemadura fue diagnosticada de acuerdo con su presentación clínica y clasificada en tres categorías: quemaduras de primero, segundo y tercer grado ${ }^{5}$. La extensión de la quemadura fue determinada usando la regla de los nueve de Wallace ${ }^{1,5}$.

Se realizó una revisión de todos los pacientes ingresados a la unidad en el periodo del estudio, se seleccionaron lo que presentaban quemaduras por agentes químicos, se excluyeron los pacientes cuyas quemaduras eran secundarias a la combustión del agente pues estas son consideradas quemaduras por calor (Ver Figura 1). 


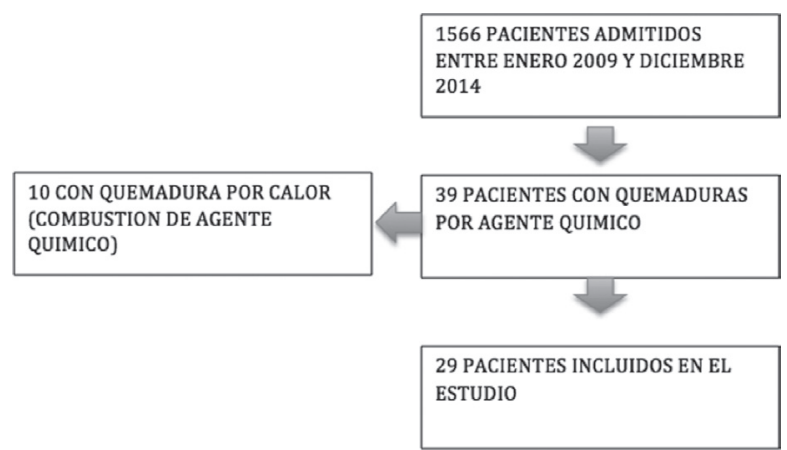

Figura 1. Flujograma para la selección de los pacientes incluidos en el estudio

Fuente: autores.

Consecutivamente se clasificaron según género, edad, extensión, profundidad, sitio anatómico comprometido y contexto de ocurrencia del trauma. Estas variables mencionadas fueron tabuladas y mostradas en las diferentes tablas y figuras.

Para el análisis de los datos se utilizó el programa estadístico de Microsoft Excel $2014^{\circledR}$, y para la presentación de los resultados se usaron porcentajes, medidas de tendencia central (promedios) y de dispersión (rangos).

\section{ResultadOS}

La Unidad de Quemados del HUS recibió un total de 29 pacientes con quemaduras químicas con un promedio anual de 5 pacientes, lo que representa el $1,5 \%$ del total de pacientes quemados que son atendidos en esta unidad por año. Del total de pacientes el $59 \%(n=17)$ son hombres y el $41 \%(n=12)$ mujeres. Durante el último año del estudio se presentó un incremento en la incidencia de quemaduras por este mecanismo (Ver Figura 2).

=Género Masculino =Género Femenino

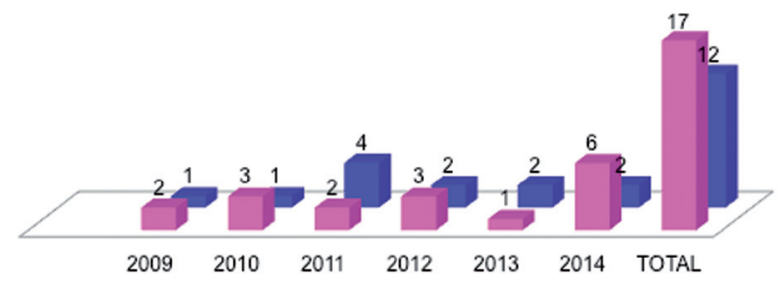

Figura 2. Distribución de ingresos hospitalarios por número de casos año y género de las quemaduras químicas 2009-2014 en la Unidad de quemados del HUS Fuente: autores.

Al discriminar la población atendida de acuerdo a los rangos de edad se encontró que el $76 \%(n=22)$ correspondían a población mayor de 18 años y el
$24 \%(n=7)$ a menores de edad, entre los cuales el $17 \%(n=5)$ son menores de 5 años de edad. (Ver Figura 3).

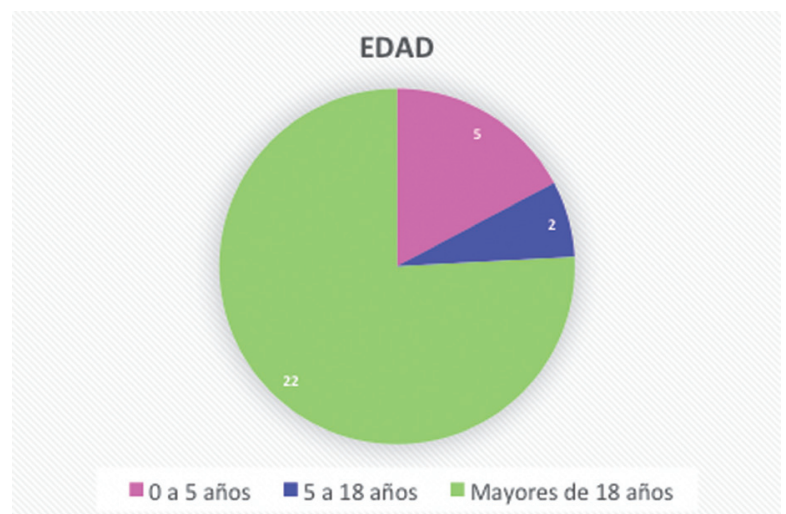

Figura 3. Clasificación por rangos de edades de las quemaduras químicas 2009-2014 en la Unidad de quemados del HUS Fuente: autores.

Dentro de los agentes químicos implicados, el grupo que mayor número de casos presentó son los ácidos $59 \%(n=17)$, dentro de los cuales en el $21 \%(n=6)$ no se logró especificar el tipo sustancia determinándose que se trataba de un ácido por las características clínicas de la escara; $21 \%(n=6)$ se debieron a ácido sulfúrico, sustancia altamente corrosiva de uso común en la industria como un potente disolvente; un total de $10 \%(n=3)$ correspondió al ácido clorhídrico cuyo nombre popular es ácido muriático y es muy usado para labores de desinfección doméstica; el porcentaje restante corresponde ácido cítrico $3 \%(n=1)$ y ácido tricloroacético 3\% $(n=1)$. En segundo lugar se encuentran los álcalis con el $28 \%(n=8)$, donde la sustancia representativa es el hidróxido de sodio, comúnmente conocida como soda cáustica la cual es utilizada en la fabricación de papel, tejidos y detergentes en labores industriales y a nivel doméstico son reconocidas sus utilidades para desbloquear tuberías de desagües de cocinas y baños. Las demás correspondieron a sustancias inorgánicas, $6 \%(n=2)$ por hipoclorito, $3 \%(n=1)$ por nitrato de potasio y $3 \%(n=1)$ por sulfato de magnesio (Ver Figura 4).

Referente al contexto donde ocurrieron las lesiones, la mayoría de los casos corresponden a accidentes laborales 55\% $(n=16)$, seguido de accidentes domésticos $28 \%(n=8)$ y $17 \%(n=5)$ por agresión. Dentro de las lesiones por agresión el $80 \%(n=4)$ correspondieron al género femenino y $20 \%(n=1)$ al masculino (Ver Figura 4 y 5$)$. 


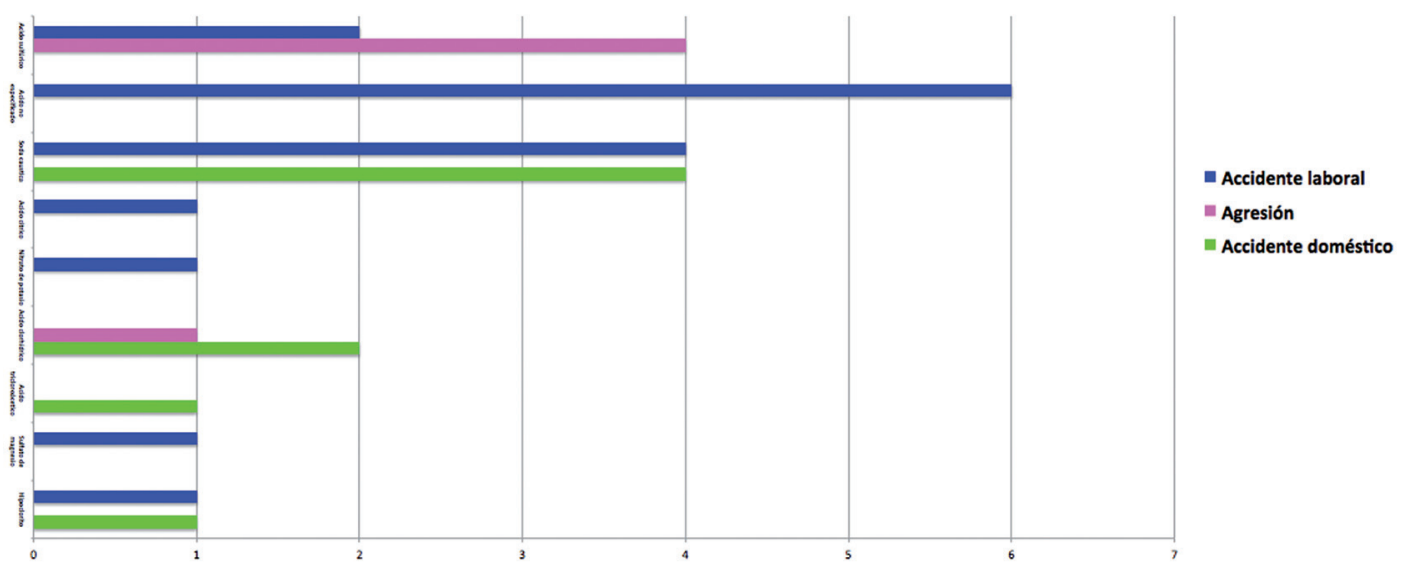

Figura 4. Agentes causales y contextos involucrados en las quemaduras químicas 2009-2014 en la Unidad de quemados del HUS Fuente: autores.

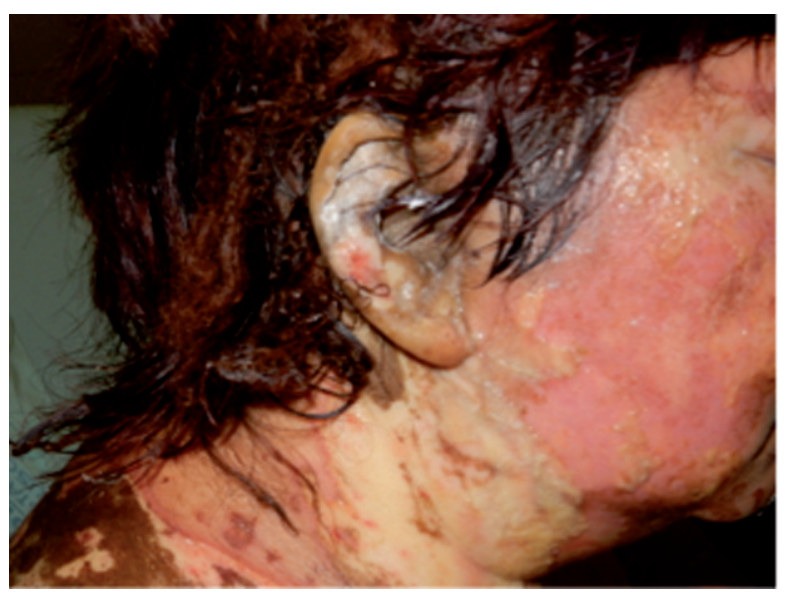

Figura 5. Mujer de 39 años con quemadura por agresión con ácido clorhídrico, grado II y grado III que comprometen el $5 \%$ de la superficie corporal total en cara y cuello Fuente: autores.

En relación a la extensión de la quemadura ninguno de los pacientes presentaba un compromiso de la superficie corporal mayor al 25\% (Ver Tabla 1). Respecto a la profundidad, la mayoría de quemaduras eran de segundo grado, seguidas por las de tercer grado (Ver Tabla 2), y alrededor de una quinta parte de ellas correspondían a quemaduras mixtas (segundo y tercer grado) (Ver Figura 6).

Tabla 1. Porcentaje de superficie corporal total comprometida en las quemaduras químicas 2009-2014 en la Unidad de Quemados del HUS

\begin{tabular}{|c|c|c|}
\hline $\begin{array}{c}\text { Porcentaje de } \\
\text { quemadura }\end{array}$ & $\begin{array}{c}\text { Número de } \\
\text { pacientes }\end{array}$ & $\begin{array}{c}\text { Porcentaje } \\
\text { de pacientes }\end{array}$ \\
\hline $0-5 \%$ & 12 & $41 \%$ \\
$5-10 \%$ & 6 & $21 \%$ \\
$10-15 \%$ & 7 & $24 \%$ \\
$15-25 \%$ & 4 & $14 \%$ \\
\hline Total & 29 & $100 \%$ \\
\hline
\end{tabular}

Fuente: autores.
Tabla 2. Profundidad de las quemaduras químicas de los pacientes admitidos en la Unidad de Quemados del HUS entre los años 2009 a 2012

\begin{tabular}{|c|c|c|}
\hline Profundidad & Número de pacientes & Porcentaje \\
\hline Grado II & 12 & $41 \%$ \\
\hline Grado II-III & 6 & $21 \%$ \\
\hline Grado III & 11 & $38 \%$ \\
\hline Total & 29 & $100 \%$ \\
\hline
\end{tabular}

Fuente: autores.

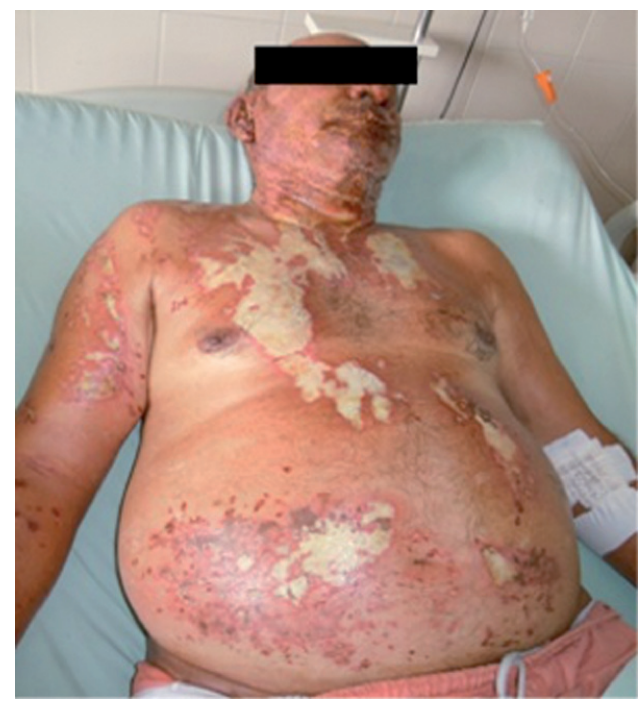

Figura 6. Paciente masculino de 80 años de edad con quemaduras grado II y III que comprometen el $15 \%$ de la superficie corporal total por accidente laboral con ácido sulfúrico Fuente: autores.

Según el sitio anatómico comprometido el lugar más frecuentemente involucrado es el miembro superior $28 \%(n=8)$, en segundo lugar la cara $24 \%(n=7)$, seguidos por el región anterior del tórax 21\% $(n=6)$, miembro inferior $17 \%(n=5)$ casos y región posterior del tórax $10 \%(n=3)$ casos. 


\section{ERERO-ABRIL}

En referencia al manejo, la totalidad ameritó tratamiento quirúrgico para realizar lavado y desbridamiento de las quemaduras, el $65 \%(n=19)$ de los pacientes se llevaron a un segundo tiempo quirúrgico para realizar cubrimiento cutáneo con injertos o colgajos, mientras que el restante recibió manejo conservador con curaciones presentando reepitelización espontánea.

\section{Discusión}

Este es el primer reporte sobre quemaduras químicas realizado en Colombia, cuyos resultados son semejantes a los encontrados en revisiones sistemáticas de la literatura como el estudio de Hardwicke y colaboradores en Reino Unido ${ }^{3}$. Se evidencia que no existen diferencias significativas en cuanto a la epidemiologia de las quemaduras químicas con respecto a la de otras unidades de quedamos en el mundo: predominio en el género masculino, mayor compromiso en edades productivas de la vida y extensiones que no superan el $15 \%$ de superficie corporal total, sin embargo la mayoría de ellas graves por profundidad y requerimiento de manejo quirúrgico ${ }^{3,6-13}$. Respecto al contexto en que ocurre el trauma, al igual que en otros estudios ${ }^{6,8-10}$ la mayoría están relacionadas con el desarrollo de actividades de tipo industrial lo cual podría reflejar deficiencia en la capacitación y control de los trabajadores que manipulan este tipo de sustancias ${ }^{3,10-13}$; un número considerable de casos se relacionan con el uso de sustancias químicas de libre expendio usadas en labores domésticas ${ }^{6,7}$ lo que alerta sobre la necesidad de regulación de su comercio con el fin de prevenirlas.

Se encontró que el sitio anatómico más frecuentemente comprometido son los miembros superiores y la cara, lo que podría reflejar una relación directa entre manipulación inadecuada y la ocurrencia de estos eventos ${ }^{3,6,8-11}$. Los agentes más comúnmente implicados son los ácidos seguido del hidróxido de sodio lo cual también se podría relacionar con el mayor uso del primero en el contexto industrial y del segundo en el doméstico ${ }^{3,6-13}$.

En cuanto a las quemaduras químicas en niños, en concordancia con el estudio de D'Cruz y colaboradores $^{14}$, la mayoría de los niños se encontraban en edades inferiores a los cinco años y la totalidad de los casos ocurrieron en el contexto doméstico por manipulación accidental, se han

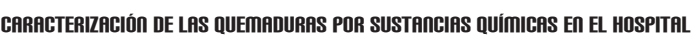

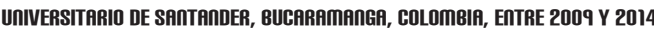

descrito un número considerable de casos cuyo mecanismo principal es la ingestión ${ }^{14,15}$, sin embargo en este estudio no se encontró ningún caso de quemadura por ingestión.

Es de resaltar que hasta antes del año 2011 países como Pakistán y Bangladesh eran lo que mayores tasas de personas quemadas con sustancias químicas por agresión registraban ${ }^{16}$, sin embargo para el periodo comprendido entre el 2011 al 2012 Colombia paso a ocupar el primer lugar a nivel mundial pasando de una tasa de 1,97 a 3,98 mujeres víctimas de agresiones por agentes químicos por cada millón de habitantes ${ }^{17}$. En este estudio se encontró una incidencia constante de un caso por año, no se encontró aumento en el número de casos en el periodo de mayor incidencia en el país, lo que contrasta con el estudio de Gaviria y colaboradores, en la unidad de quemados del Hospital Simón Bolívar, en Bogotá18.

\section{CONCLUSIONES}

Los resultados obtenidos reflejan que las quemaduras químicas se asocian a situaciones accidentales donde predomina el desconocimiento del potencial lesivo de estas sustancias llevando a su manipulación negligente y daño secundario, lo que plantea un reto para la elaboración de campañas de prevención primaria, como la capacitación a los trabajadores sobre bioseguridad industrial para el uso elementos protectores como trajes, guantes y tapabocas; y a su vez refleja la necesidad de regulación sobre el libre expendio de dichas sustancias, el cual debería limitarse a personas capacitadas en su correcta manipulación.

El presente trabajo se convierte en una referencia para contrastar futuros estudios publicados en otras unidades de quemados a fin de conocer el panorama general y determinar necesidades en cuanto a prevención primaria, manejo y prevención secundaria.

\section{Consideraciones ÉtICAS}

La confidencialidad de los datos y la identidad de los pacientes fueron resguardadas con el debido consentimiento informado previamente solicitado durante la atención hospitalaria a través de un formato estándar institucional en el cual se incluye la divulgación de los presentes datos y fotografías con fines académicos. 


\section{CONFLICTO DE INTERÉSES}

Los autores del presente trabajo no tienen ningún tipo de conflicto de interés ni subvención para la realización y la publicación de este manuscrito.

\section{AGRADECIMIENTOS}

Al médico y cirujano Jairo A. Sierra-Avendaño, por su contribución en el diseño del estudio, presentación de los datos y la revisión del manuscrito final. Al ingeniero industrial John Gonzales, por su ayuda en la tabulación y graficación de los datos.

\section{REFERENCIAS BIBLIOGRÁFICAS}

1. Ramírez-Rivero CE, Ramírez-Blanco CE, Ramírez-Blanco N, Ramírez-Blanco MA. Manejo del paciente quemado. Rev. Univ. Ind. Santander. Salud. 2007;39(2):124-136.

2. Palao R, Monge I, Ruiz M, Barret JP. Chemical burns: Pathophysiology and treatment. Burns. 2010;36(3):295-304.

3. Hardwicke J, Hunter T, Staruch R, Moiemen N. Chemical burns-an historical comparison and review of the literature. Burns 2012;38(3):383-7.

4. Ramirez-Rivero CE, Ramírez-Blanco CE, Ramírez N, Butz DR. Epidemiología de la Unidad de Quemados del Hospital Universitario de Santander. RCCP. 2008;14(1):15-23. bien

5. Ramírez-Rivero C, Ramírez-Blanco C, Ramìrez-Blanco MA, Ramírez-Blanco N. Fisiopatología del paciente quemado. Rev. Univ. Ind. Santander. Salud. 2010;42(1):55-65.
6. Pitkanen J, Al-Qattan MM. Epidemiology of domestic chemical burns in Saudi Arabia. Burns. 2001;27(4):376-8.

7. Iribarren B. Osvaldo, Gonzales G. Claudio. Quemaduras por agentes químicos. Cuad. cir. (Valdivia). 2001;15(1):61-69.

8. Robinson EP, Chhabra AB. Hand chemical burns. J Hand Surg. Am. 2015;40(3):605-12; quiz 613.

9. Ricketts S, Kimble FW. Chemical injuries: The Tasmanian Burns Unit experience. ANZ J Surg. 2003;73(1-2):45-8.

10. Wei Li, Xiaofeng Wu, Chengjin Gao. Ten-year epidemiological study of chemical burns in Jinshan, Shanghai, PR China. Burns. 2013;39(7):1468-73.

11. Ramakrishnan K.M, Mathivanan T, Jayaraman V, Babu M, Shankar J. Current scenario in chemical burns in a developing country: Chennai, india. Annals of Burns and Fire Disasters. 2012;15(1):8-12.

12. Hemmat Maghsoudi, Nasibeh Gabraely. Epidemiology and outcome of 121 cases of chemical burn in East Azarbaijan province, Iran. Injury. 2008;39(9):1042-6.

13. Youfu Xie a. Yanyan Tan a. Shuze Tang. Epidemiology of 377 patients with chemical burns in Guangdong province. Burns. 2004;30:569-572.

14. D'Cruz R. Pang TCY. Harvey JG. Holland AJA. Chemical burns in children: Aetiology and prevention. Burns. 2015;41:764-9.

15. Kay M. Wyllie R. Caustic ingestions in children. Curr Opin Pediatr. 2009;21(5):651-4.

16. Mannan A. Ghani S. Clarke A. Butler PEM. Cases of chemical assault worldwide: a literature review. Burns. 2007;33(2):149-54.

17. Protocolo de Atención Integral de Urgencias a Víctimas de Agresiones por Químicos. Abril de 2014. Disponible en: http:// www.consultorsalud.com/sites/consultorsalud/files/Protocolo\%2 520 atencion\%2520urgencias\%2520ataques\%2520quimicos\%25 202014\%2520Minsalud.pdf

18. Gaviria-Castellanos JL. Gómez-Ortega V. Gutiérrez P. Quemaduras químicas por agresión: características e incidencia recogidas en el Hospital Simón Bolívar, Bogotá, Colombia. Cir Plástica Ibero Latinoam. 2015;41(1):73-82.

19. Guerrero L. Burns due to acid assaults in Bogotá, Colombia. Burns. 2013;39(5):1018-23. 